\title{
Corneal Collagen Cross-Linking Mushroom Shape Demarcation Line Profile After Limited Bowman's Membrane Removal by Phototherapeutic Keratectomy
}

\author{
George D. Kymionis ${ }^{*}{ }^{, 1,2}$, Michael A. Grentzelos ${ }^{1}$, Nektarios E. Klados ${ }^{1}$, Niki A. Xanthopoulou ${ }^{1}$, \\ Theodore A. Paraskevopoulos ${ }^{1}$ and Efstathios T. Detorakis \\ ${ }^{I}$ Vardinoyiannion Eye Institute of Crete (VEIC), Faculty of Medicine, University of Crete, Heraklion, Crete, Greece \\ ${ }^{2}$ Bascom Palmer Eye Institute, Miller School of Medicine, University of Miami, Miami, FL, USA
}

\begin{abstract}
Purpose: To report a corneal collagen cross-linking (CXL) mushroom shape demarcation line profile after limited Bowman's membrane removal by phototherapeutic keratectomy (PTK).

Methods: Case report.

Results: A twenty-one-year-old male with progressive keratoconus underwent mechanical epithelial debridement (at an $8.5 \mathrm{~mm}$ zone) followed by PTK (at a $5.0 \mathrm{~mm}$ zone and in a $10 \mu \mathrm{m}$ depth) and CXL. No intra- or early postoperative complications were found. Evaluation of the corneal stromal demarcation line depth using anterior segment optical coherence tomography revealed a mushroom shape profile.

Conclusion: It seems that removal of the Bowman's layer leads to greater depth of the corneal stromal demarcation line.
\end{abstract}

Keywords: Cross-linking, keratoconus, phototherapeutic keratectomy.

\section{INTRODUCTION}

Excimer laser phototherapeutic keratectomy (PTK) is a surgical procedure that has been applied for the treatment of anterior corneal diseases, including anterior corneal dystrophies, degenerations, recurrent corneal erosions and corneal scars [1]. Corneal collagen cross-linking (CXL) is a surgical method, which is used to increase corneal rigidity and halt the keratoconus progression [2]. Phototherapeutic keratectomy is combined with corneal collagen cross-linking in order to improve the visual and refractive outcomes of CXL [3-5]. After application of CXL, anterior stromal alterations caused by the treatment produce a corneal stromal demarcation line, which is identified clinically in slit-lamp examination, or with the use of imaging techniques, such as confocal microscopy and anterior segment optical coherence tomography (AS-OCT) [6-10].

In the current article, we report a case of a patient with keratoconus, who was treated with mechanical epithelial debridement (at an $8.5 \mathrm{~mm}$ zone) followed by limited PTK (at a $5.0 \mathrm{~mm}$ zone and in a $10 \mu \mathrm{m}$ depth) and CXL. AS-OCT revealed a mushroom shape demarcation line profile indicating the possible role of the Bowman's layer removal for greater corneal stromal demarcation line depth.

*Address correspondence to this author at the University of Crete, Faculty of Medicine, Institute of Vision and Optics (IVO), 71003 Heraklion, Crete, Greece; Tel: +302810371800; Fax: +302810394653;

E-mail: kymionis@med.uoc.gr

\section{CASE REPORT}

A twenty-one-year-old male patient with topographically confirmed progressive keratoconus presented to our institute for consultation. During examination, his uncorrected distance visual acuity (UDVA) was 20/63 in the right and $20 / 250$ in the left eye, while corrected distance visual acuity (DCVA) was 20/20 in both eyes. Keratometric readings were 46.13 / 42.40 and 43.62 / 42.82 in the right and the left eyes, respectively. Corneal topography revealed a centrally located corneal cone configuration. The corneal pachymetric values, estimated with the use of ultrasound, were $472 \mathrm{~nm}$ in the right eye, and $504 \mathrm{~nm}$ in the left eye. The keratoconus was graded as stage 2, according to the Amsler - Krumeich classification. Slit-lamp examination of anterior segment and fundoscopy did not reveal any pathological features. The patient underwent uneventful CXL treatment in his right eye.

\section{SURGICAL TECHNIQUE}

After instillation of proxymetacaine hydrochloride $0.5 \%$ eyedrops (Alcaine; Alcon Laboratories, Inc, Fort Worth, Texas, USA) as a topical anaesthetic, corneal epithelium debridement was achieved with the use of a rotating brush at an $8.5 \mathrm{~mm}$ zone. After mechanical epithelial removal, PTK was performed at a $5.0 \mathrm{~mm}$ zone in a $10 \mu \mathrm{m}$ depth. After PTK, riboflavin $(0.1 \%$ solution of $10 \mathrm{mg}$ riboflavin-5phosfate in $10 \mathrm{ml}$ dextran-T-500 $20 \%$ solution, Medicross, Medio - Haus, Behrensbrook, Neudorf, Germany) was applied repeatedly on the central cornea every 3 minutes for a duration time of approximately 30 minutes. The cornea 


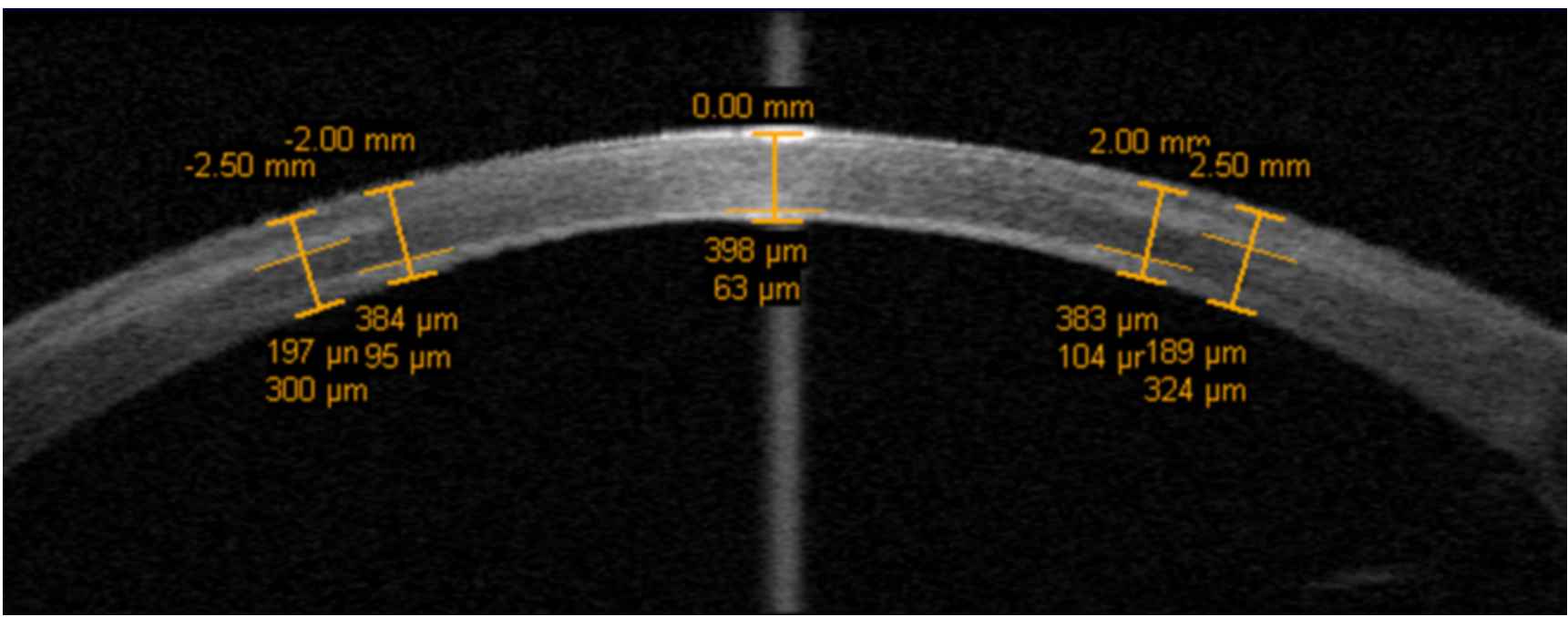

Fig. (1). High resolution anterior segment corneal optical coherence tomography scan demonstrating the corneal stromal demarcation line one month after mechanical epithelial debridement followed by phototherapeutic keratectomy and corneal collagen cross-linking in a patient with progressive keratoconus with depths of $398 \mu \mathrm{m}$ (centrally), $384 \mu \mathrm{m}$ and $383 \mu \mathrm{m}$ (at $2.0 \mathrm{~mm}$ nasally and temporally, respectively), 197 $\mu \mathrm{m}$ and $189 \mu \mathrm{m}$ (at $2.5 \mathrm{~mm}$ nasally and temporally, respectively).

was irradiated with Ultraviolet-A (UV-A) illumination with the use of an UV-A optical device (CCL-365, Peschke Meditrade GmbH, Huenenberg, Switzerland). Before treatment, the device was calibrated using the UV-A light meter YK-35UV (Lutron Electronic, Coopersburg, PA), which is supplied with the UV-X device, in order to ensure that the irradiance was $3.0 \mathrm{~mW} / \mathrm{cm}^{2}$, as intended. Total irradiance time was 30 minutes, as required in order to administer a total surface dose of $5.4 \mathrm{~J} / \mathrm{cm}^{2}$. During UV-A irradiation procedure, riboflavin solution instillation was continued repeatedly every 3 minutes to maintain corneal saturation with riboflavin. At the end of the procedure, a silicone-hydrogel (Lotrafilcon B, Air Optix, Ciba Vision $14.0 \mathrm{~mm}$ diameter, 8.6 base curvature, $\mathrm{Dk}=140$ barrers) bandage contact lens (BCL) was applied until complete epithelium healing.

One month after the treatment CXL procedure, UDVA was improved at 20/40 and CDVA remained stable at 20/20. Slit-lamp biomicroscopy revealed a clear cornea. Anterior segment optical coherence tomography (AS-OCT) was performed in order to identify and measure the depth of the corneal stromal demarcation line depth produced by CXL treatment. Corneal stromal demarcation line depth was measured centrally, at $2.0 \mathrm{~mm}(4.0 \mathrm{~mm}$ diameter $)$ and at 2.5 $\mathrm{mm}$ (5.0 $\mathrm{mm}$ diameter) nasally and temporally, as described in our previous study [9]. Corneal stromal demarcation line depth was $398 \mu \mathrm{m}$ centrally, $384 \mu \mathrm{m}$ and $383 \mu \mathrm{m}$ at $2.0 \mathrm{~mm}$, $197 \mu \mathrm{m}$ and $189 \mu \mathrm{m}$ at $2.5 \mathrm{~mm}$, nasally and temporally, respectively.

\section{DISCUSSION}

Excimer laser PTK is a surgical procedure that has been successfully applied for the treatment of anterior corneal pathology, including anterior corneal dystrophies, degenerations corneal scars and recurrent corneal erosions [1]. CXL is a surgical method used to increase the corneal rigidity, stabilize the ectatic cornea and arrest the keratoconus progression [2]. PTK is combined with CXL in order to remove the epithelium, smoothen the anterior corneal surface and decrease the irregular astigmatism induced by the keratoconus [3-5].

Two weeks after CXL, a corneal stromal demarcation line can be identified clinically during slit-lamp examination in a depth of approximately $300 \mu \mathrm{m}$ [6], or with the use of imaging modalities, such as confocal microscopy and ASOCT representing the depth of the CXL treatment [7-10]. Recently, we showed that both confocal microscopy and AS OCT have similar accuracy in the evaluation of the depth of the corneal stromal demarcation line produced with CXL treatment [10].

In the current case report, a patient with progressive keratoconus is presented, who underwent mechanical epithelial debridement followed by limited PTK and CXL. The aim of combining PTK with CXL at the given size was to demonstrate the increase in treatment depth of CXL after Bowman's layer removal. One month postoperatively, ASOCT revealed a mushroom demarcation line profile (Fig. 1); corneal stromal demarcation line depth was greater centrally and at $2.0 \mathrm{~mm}$ (nasally and temporally) than at $2.5 \mathrm{~mm}$ (nasally and temporally).

A possible explanation of this configuration could be the Bowman's membrane removal. Kolozsvari et al. have shown that Bowman's layer absorb high amounts of UV irradiation [11]. Therefore, it seems that removal of the Bowman's layer in our patient increased UV-A penetration in the corneal stroma leading to greater corneal stromal demarcation line depth in the PTK ablated central corneal area. In addition, it is possible that Bowman's removal may induce corneal thinning through enhanced evaporation. Even though the role of Bowman's layer in the CXL procedure is unknown this finding indicates indirectly that Bowman's layer removal could increase the depth of CXL treatment.

\section{CONFLICT OF INTEREST}

The authors confirm that this article content has no conflict of interest. 


\section{ACKNOWLEDGEMENTS}

Declared none.

\section{REFERENCES}

[1] Rapuano CJ. Excimer laser phototherapeutic keratectomy. Curr Opin Ophthalmol 2001; 12: 288-93.

[2] Wollensak G, Spoerl E, Seiler T. Riboflavin/ultraviolet-A-induced collagen crosslinking for the treatment of keratoconus. Am J Ophthalmol 2003; 135: 620-7.

[3] Kymionis GD, Grentzelos MA, Kounis GA, Diakonis VF, Limnopoulou AN, Panagopoulou SI. Combined transepithelial phototherapeutic keratectomy and corneal collagen cross-linking for progressive keratoconus. Ophthalmology 2012; 119: 1777-84.

[4] Kymionis GD, Grentzelos MA, Karavitaki AE, et al. Transepithelial phototherapeutic keratectomy using a 213-nm solidstate laser system followed by corneal collagen cross-linking with riboflavin and UVA irradiation. J Ophthalmol 2010; 2010: 146543.

[5] Kymionis GD, Grentzelos MA, Kankariya VP, et al. Long-term results of combined transepithelial phototherapeutic keratectomy and corneal collagen crosslinking for keratoconus: Cretan protocol. J Cataract Refract Surg 2014; 40(9): 1439-45.
[6] Seiler T, Hafezi F. Corneal cross-linking-induced stromal demarcation line. Cornea 2006; 25: 1057-9.

[7] Mazzotta C, Balestrazzi A, Traversi C, et al. Treatment of progressive keratoconus by riboflavin-UVA-induced crosslinking of corneal collagen: ultrastructural analysis by Heidelberg Retinal Tomograph II in vivo confocal microscopy in humans. Cornea 2007; 26: 390-7.

[8] Doors M, Nayyirih G. Tahzib, et al. Use of anterior segment optical coherence tomography to study corneal changes after collagen cross-linking. Am J Ophthalmol 2009; 148: 844-51.

[9] Kymionis GD, Grentzelos MA, Plaka AD, et al. Evaluation of the corneal collagen cross-linking demarcation line profile using anterior segment optical coherence tomography. Cornea $2013 ; 32$ 907-10.

[10] Kymionis GD, Grentzelos MA, Plaka AD, et al. Correlation of the corneal collagen cross-linking demarcation line using confocal microscopy and anterior segment optical coherence tomography in keratoconic patients. Am J Ophthalmol 2013; [Epub ahead of print].

[11] Kolozsvári L, Nógrádi A, Hopp B, Bor Z. UV absorbance of the human cornea in the 240- to 400-nm range. Invest Ophthalmol Vis Sci 2002; 43: 2165-8.

(C) Kymionis et al.; Licensee Bentham Open

This is an open access article licensed under the terms of the Creative Commons Attribution Non-Commercial License (http://creativecommons.org/licenses/by-nc/3.0/) which permits unrestricted, non-commercial use, distribution and reproduction in any medium, provided the work is properly cited. 